\title{
Generation of higher harmonic internal waves by oscillating spheroids
}

\author{
Natalia Shmakova, ${ }^{1, *}$ Evgeny Ermanyuk, ${ }^{1,2,3}$ and Jan-Bert Flór ${ }^{1, \dagger}$ \\ ${ }^{1}$ Laboratoire des Écoulements Géophysiques et Industriels (LEGI), CNRS-Université Grenoble Alpes, \\ F38000, Grenoble, France \\ ${ }^{2}$ Lavrentyev Institute of Hydrodynamics, Siberian Branch of the Russian Academy of Sciences, \\ Prospekt Lavrentyev 15, Novosibirsk 630090, Russia \\ ${ }^{3}$ Novosibirsk State University, Pirogova Street 2, Novosibirsk 630090, Russia
}

\begin{abstract}
Oscillating bodies in stratified fluids may emit higher harmonics in addition to fundamental waves. In the present experimental study, we consider higher harmonics of an internal wave field generated by a horizontally oscillating spheroid in a linearly stratified fluid for moderate to high oscillation amplitudes, i.e., scaled oscillation amplitude $A / a \geqslant 0.5$, with $a$ the minor radius of the spheroid. Three different spheroid shapes are tested. The results are discussed in the context of the different theories on the generation of higher harmonics. Higher harmonics are observed at the intersections of fundamental wave beams, and at the critical points of the topography where the topographic slope equals the wave slope. The velocity amplitudes of the fundamental, second, and third harmonic waves grow respectively linearly, quadratically, and with the third power of the scaled oscillation amplitude $A / a$. Though these amplitudes are generally higher when the object's slope is larger, the increase in amplitude above and below the axisymmetric oscillating objects is found to be due to the effect of focusing. In order to discern the relative importance of the harmonics to the fundamental wave, the horizontal structure of the wave amplitude is measured. The results suggest that the $n$th harmonic of the internal wave field is associated with a radiation diagram corresponding to a multipole of order $2^{n}$, with $2 n$ directions of propagation.
\end{abstract}

\section{INTRODUCTION}

In the Earth's oceans, internal waves generated by the barotropic tide are known to play an important role for its energy balance [1-4]. Motivated by this application, the oscillation of idealized bodies of different shapes in a linearly stratified fluid has been investigated in a large number of studies. Seen from the reference frame of the fluid, wave generation by an oscillating body in the linear regime is, to a good approximation, comparable to that of the interaction of the barotropic tide with bottom topography [5,6]. Most well known are the classical experiments of Mowbray and Rarity [7], which show that the dispersion relation for internal waves generated by a cylinder, oscillating with frequency $\omega$ in a linearly stratified fluid with buoyancy frequency $N$, admits for the propagation of an $n$th harmonic component [7] of the wave field if $n \omega<N$. Linear wave theory for elliptic cylinders [8,9] adequately predicts the observed fundamental wave [10], but higher harmonics seem to be due to fully nonlinear dynamics as shown by Sutherland and Linden [11] and Zhang et al. [12]. Interaction between fundamental waves and higher harmonics lead to a complex fine-scale structure over the entire fluid depth, as has been shown numerically for a two-dimensional tidal motion interacting with a mountain ridge [13].

Higher harmonics also have a three-dimensional distribution of wave amplitudes in the azimuthal direction, when generated by a three-dimensional object. For a spherical geometry, linear theory for

\footnotetext{
*shmakova@hydro.nsc.ru

†jan-bert.flor@legi.cnrs.fr
} 


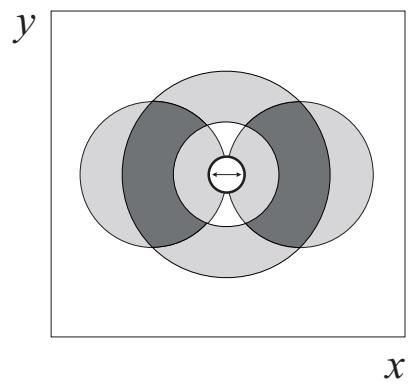

(a)

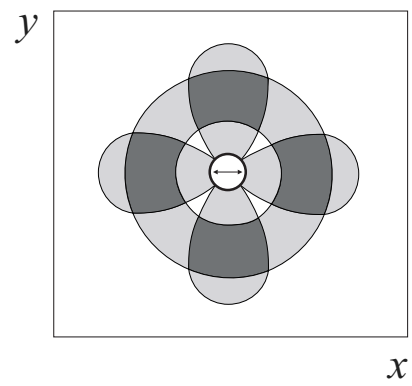

(b)

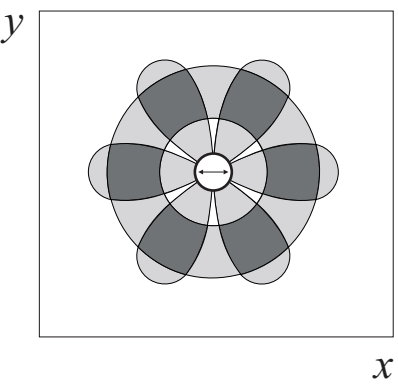

(c)

FIG. 1. Fundamental wave (large circle) superposed on the horizontal structure of the (a) dipolar, (b) quadrupolar, and (c) octupolar wave patterns. The annular propagation of the waves and the $2^{n}$-polar radiation pattern as $\cos (n \phi)$, where $\phi$ is the azimuthal angle, is represented by light gray and the combination of the fundamental wave and higher harmonic is shown in dark gray.

the fundamental wave has been described and experimentally verified [14]. A detailed study of the radiation pattern of the propagative first and second harmonics (see Ermanyuk et al. [15]) showed that first and second harmonics have dipolar and quadrupolar radiation patterns, respectively. The shapes of these patterns are shown in Fig. 1 together with the octupolar radiation pattern which we might expect for the third harmonic wave. The presence of higher harmonics has also been demonstrated for an isolated Gaussian hill by King et al. [16].

The aim of the present paper is to consider the generation of higher harmonic internal waves near topography of different elongation, here represented by an oscillating sphere, and a prolate spheroid, oriented either horizontally or vertically. Experimental observations are compared with the theoretical predictions of Bell [17] for waves on curved boundaries and Tabaei et al. [18] and Jiang and Marcus [19] for wave generation at wave beam intersections. The measurements of the horizontal flow field allow us to make a hypothesis about the horizontal structure of the $n$th harmonic radiation patterns.

Our paper is organized as follows. The experimental setup, measurement techniques, and the filtering methods used to visualize a specific higher harmonic wave are detailed in the next section. In Sec. III, the results obtained for the vertical and horizontal wave structure, wave amplitude, and time-frequency evolution are discussed in light of the theoretical results mentioned above, followed by brief conclusions in Sec. IV.

\section{EXPERIMENTAL SETUP AND DATA PROCESSING}

Experiments were conducted in an acrylic Plexiglass cubical tank with working depth of $97 \mathrm{~cm}$ and horizontal dimensions of $97 \times 97 \mathrm{~cm}^{2}$, filled to a depth of $90 \mathrm{~cm}$ with a linearly stratified fluid [see Figs. 2(a) and 2(b)]. Salt was used as the stratifying agent and tap water as the working fluid. The kinematic viscosity of this fluid, taking into account its salinity and temperature, was $v=1.1 \mathrm{~mm}^{2} / \mathrm{s}$. The stratification was measured by taking density samples at different heights in the fluid. A least-squares fit provided the density gradient and the related value of the buoyancy frequency $N(z)=[-(g / \rho)(d \rho / d z)]^{1 / 2}=$ const, with $g$ being the gravity acceleration and $\rho(z)$ being the density distribution with the vertical coordinate $z$. In the present experiments, the stratification was varied between 0.8 and $1.08 \mathrm{rad} / \mathrm{s}$.

Waves were generated by the horizontally oscillating acrylic Plexiglass spheroid of equatorial radius $a$ and polar radius $b$, attached to a pendulum of length $1.3 \mathrm{~m}$. The aspect ratio for the spheroids was $b / a=1$ (sphere) and $b / a=2$ (prolate spheroid) with $a=3.125 \mathrm{~cm}$ in both cases [Figs. 2(c)-2(e)]. The prolate spheroid oscillated either with its long axis vertical [Fig. 2(d)], referred 


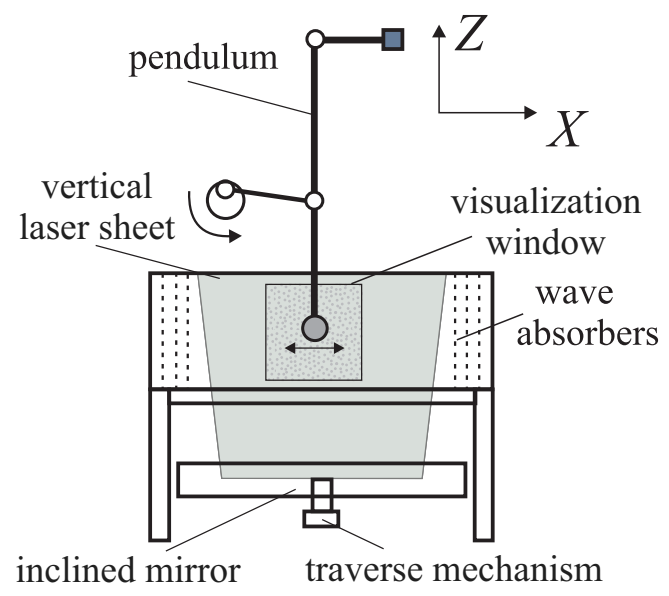

(a)

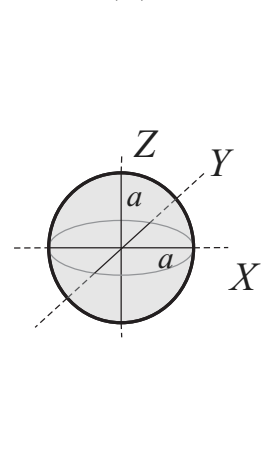

(c)

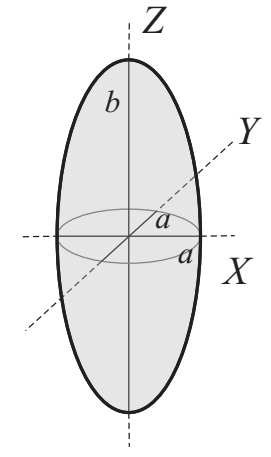

(d)

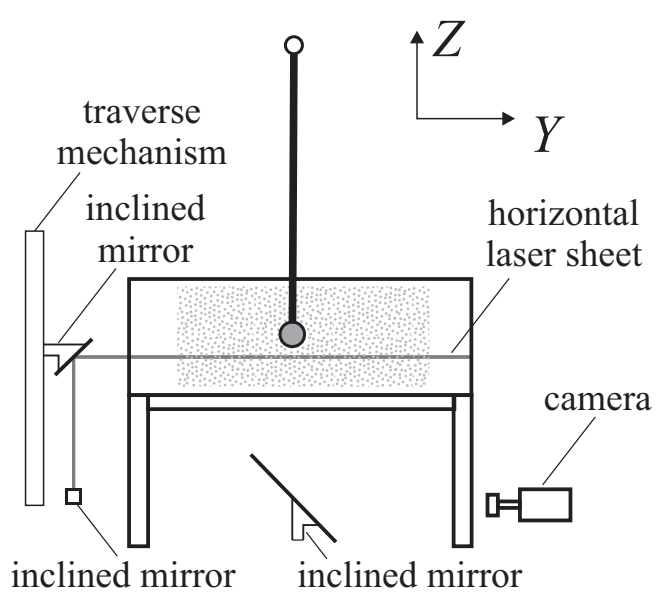

(b)

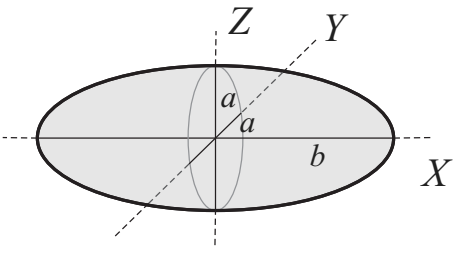

(e)

FIG. 2. Schematic views of the experimental setup: (a) front view with vertical laser plane; (b) side view with horizontal laser plane. Geometry of oscillating objects: (c) $\mathcal{S}$ sphere; prolate spheroid with (d) vertical axis of revolution $(\mathcal{V}$ spheroid) and (e) horizontal axis of revolution ( $\mathcal{H}$ spheroid).

to as $\mathcal{V}$ spheroid, or horizontal [Fig. 2(e)], referred to as $\mathcal{H}$ spheroid. The nondimensional coordinates $(X, Y, Z)=(x, y, z) / a$ are used, with the $x$ and $y$ axes respectively parallel and perpendicular to the direction of oscillation of the spheroid, as shown in Fig. 2(c) and $(x, y, z)=(0,0,0)$ at the center of the object at rest. The surface of the spheroids was painted black to avoid reflections of the laser light. The oscillations of the pendulum were driven by a crank mechanism. The motion was a good approximation horizontal and sinusoidal, with amplitude $A$ and frequency $\omega$. Wave absorbers, consisting of three layers of plastic grids of $3 \mathrm{~mm}$ thickness and square meshes of $3 \mathrm{~cm}$, were placed along the tank walls perpendicular to the plane of oscillation, effectively suppressing wave reflections. The wave pattern generally became steady after 10 oscillation periods. The same experimental apparatus has served for the experiments in Refs. [14,15].

To measure the velocity field, a two-dimensional particle image velocimetry (PIV) technique was used. The fluid was seeded with Orgasol $30-\mu \mathrm{m}$ particles of density $\rho=1.035 \mathrm{~kg} / \mathrm{m}^{3}$, which were illuminated with either a vertical or a horizontal laser sheet. The vertical laser sheet passed through the transparent bottom of the tank and was parallel to the direction of oscillation as shown in Fig. 2(a). Different vertical planes could be illuminated by moving the inclined mirror on the traverse mechanism below the tank. The position of the sheet was computer controlled and allowed for the recording of sequences of planes. For the illumination in the horizontal plane, the horizontal 
laser sheet was fixed at one height and the laser passed through the side wall of the tank as shown in Fig. 2(b).

In both cases, we measured velocities in the plane of view and assumed that the velocities perpendicular to it are small. To measure period-averaged quantities and to determine the amplitudes of the Fourier components of the signal in a vertical plane, the sheet was kept fixed during one oscillation period and then moved $0.5 \mathrm{~cm}$ to the next position, until enough vertical planes were obtained to reconstruct the vertical and horizontal longitudinal velocity components in three dimensions by spline interpolation. In the horizontal plane, long time series were taken in order to process the data with Fourier transform techniques [20,21] (and in particular with the Hilbert transform). The size of the visualization window was $36 \times 36 \mathrm{~cm}^{2}$ and $53 \times 53 \mathrm{~cm}^{2}$ for the vertical and horizontal planes, respectively. Images were recorded with a 12-bit DALSA digital camera with charge-coupled device $(\mathrm{CCD})$ matrix of $1024 \times 1024$ pixels.

In the experiments, successive images of the wave field were taken at time intervals of $\Delta t=0.2 \mathrm{~s}$. This interval was chosen to be sufficiently small compared to the period of oscillations so that the number of images per period was large, i.e., between 56 and 84. The particle displacement was obtained by cross correlating two successive images using the velocity image correlation algorithm UVMAT/CIVx [22]. Fourier decompositions of time series of the horizontal, $u(t)$, and vertical, $w(t)$, velocity were used to separate the fundamental wave and its higher harmonics. For the vertical plane, the amplitude of horizontal, $u_{a}$, and vertical, $w_{a}$, velocity was defined as root mean square of $u(t)$ and $w(t)$, respectively, multiplied by $\sqrt{2}$ [11]. The moduli $u_{a}$ and $w_{a}$ of the Hilbert transformation of $u(t)$ and $w(t)$ were taken in the horizontal plane. Nondimensional horizontal (longitudinal) and vertical velocity amplitudes are respectively given by $U_{a}=u_{a} / A \omega$ and $W_{a}=w_{a} / A \omega$.

\section{RESULTS}

In a uniformly stratified fluid, the oscillation of a body generates internal waves with their generatrices inclined at the angle

$$
\theta=\arccos \Omega
$$

to the vertical where $\Omega=\omega / N$ is the frequency ratio [23]. The dispersion relation (1) admits for the generation of a propagative $n$th harmonic component of the wave field if $n \Omega<1$. The horizontal structures of the first and the second harmonics generated by a sphere have been studied by Ermanyuk et al. [15].

The effective generation of the $n$th harmonic requires an appropriate choice of the nondimensional oscillation amplitude $A / a$ and oscillation frequency $\Omega$. Obviously, $A / a$ should be large enough for the amplitude of the $n$th harmonic, proportional to $(A / a)^{n}$, to be observable. Based on the knowledge of the behavior of the first harmonic, a plausible conjecture for the frequency tuning can be formulated. For circular cylinders and spheres oscillating with a small fixed amplitude, the most efficient transmission of energy into first harmonic waves is found for $\Omega$ between 0.7 and 0.8 (see Fig. 26 in Ref. [14]). Since the particular geometry of the wave generator has a weak influence on the frequency corresponding to the maximum radiated power, we can make the conjecture that the $n$th harmonic, irrespective of its actual generation mechanism, is most effectively generated when $0.7<n \Omega<0.8$. This condition was used for the frequency tuning in the present experiments.

\section{A. Vertical structure}

The wave patterns emitted by a horizontally oscillating prolate spheroid are shown in Fig. 3, for the $\mathcal{H}$ and the $\mathcal{V}$ spheroids in the left and right panels, respectively. This change in the orientation of the spheroid allows for the study of the interaction of vertically or horizontally elongated topography with the stratification. For both spheroids, schematic illustrations of the first and second harmonic generation is performed in Fig. 4 based on the theoretical predictions described in Tabaei et al. [18], Jiang and Marcus [19], and Bell [17]. In the left column of Fig. 3, one notices the generation of second 


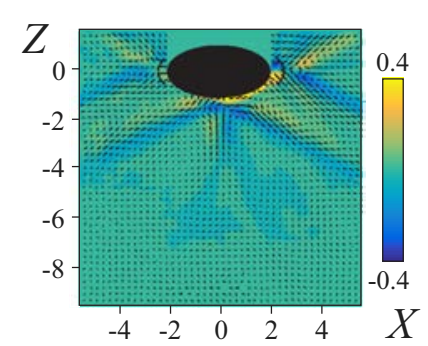

(a)

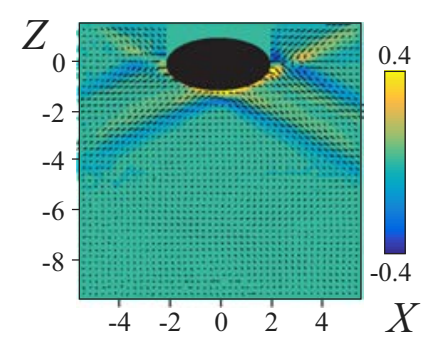

(c)

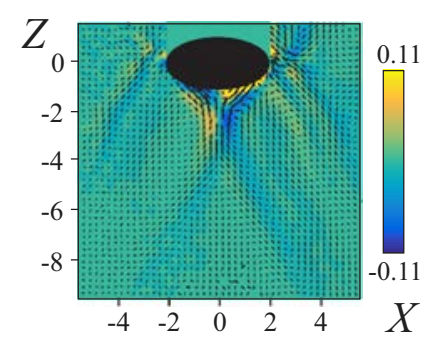

(e)

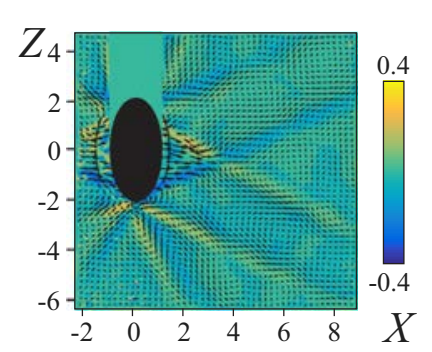

(b)

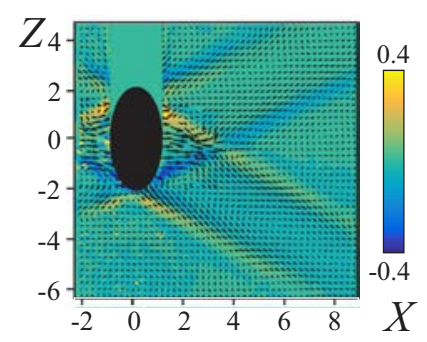

(d)

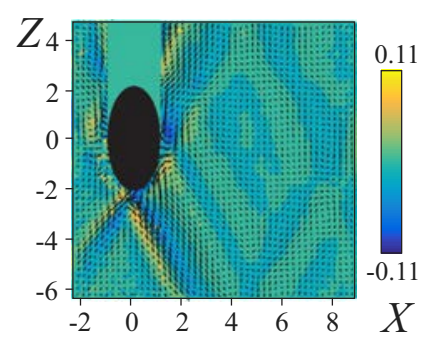

(f)

FIG. 3. Instantaneous vorticity and velocity vector field of the wave field in the vertical plane of symmetry generated by a $\mathcal{H}$ spheroid (left column) and a $\mathcal{V}$ spheroid (right column): [(a), (b)] total (fundamental and second harmonic), [(c), (d)] first harmonic, and [(e), (f)] second harmonic. Experimental parameters: $N=0.89 \mathrm{rad} / \mathrm{s}, \Omega=0.44, A / a=0.5$. Images are presented at the phase $\pm \pi / 4$ for the $\mathcal{V}$ and $\mathcal{H}$ spheroids, respectively. Dashed black lines in panels (a) and (b) correspond to extreme positions of spheroids.

harmonic waves at $Z=0$, i.e., at the intersections of the fundamental waves. In agreement with the theory of Tabaei et al. [18] and Jiang and Marcus [19], only fundamental and second harmonic waves are generated that propagate away from the obstacle when $1 / 3<\Omega<1 / 2$ [corresponding with the blue dashed lines in Fig. 4(a)]. The waves below (and above) the spheroid are generated at the boundary and appear to have their origin at the critical points, where the slope of the topography $s$ equals the slope of the harmonic wave, i.e.,

$$
\varepsilon_{i}=\frac{s}{\sqrt{\omega_{i}^{2} /\left(\omega_{i}^{2}-N^{2}\right)}}=1,
$$

$i=1,2$ for the first and the second harmonics, respectively $\left(\omega_{2}=2 \omega_{1}\right)$ [see green and red filled circles in Fig. 4(a)]. Bell's theory [17] could apply and be responsible for the emission of harmonic waves near the boundary. Second harmonic wave beams emitted from critical points $\varepsilon_{2}=1$ intersect below the spheroid and therefore locally amplify in wave energy [Fig. 3(e)]. However, the position of the focal zone does not correspond to the one predicted according to the dispersion relation $\theta=\arccos (2 \Omega)$ [see red dashed lines in Fig. 4(a)] for a second harmonic wave. The $\mathcal{H}$ spheroid is 


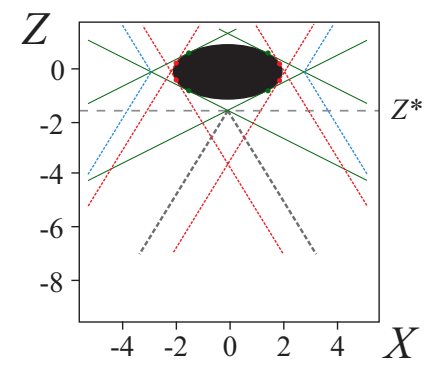

(a)

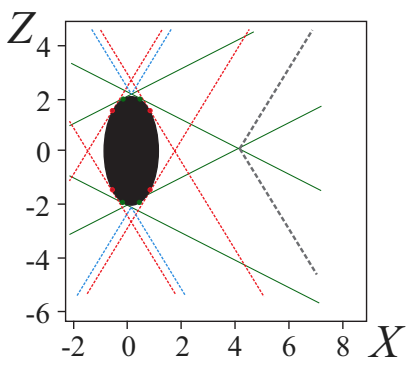

(b)

FIG. 4. Schematic representation of the first and second harmonics of waves generated (according to the theory of Refs. [17-19]) by (a) the horizontally oriented and (b) vertically oriented prolate spheroids, noted respectively as $\mathcal{H}$ and $\mathcal{V}$ spheroids in the text; green and red dots correspond to critical points $\varepsilon_{1,2}=1$, where first and second harmonics are generated. Fundamental (green lines) and second (red dashed lines) harmonic generation from critical points $\varepsilon_{i}$ at the boundary agrees with theory [17]; second harmonic generation from zones of fundamental wave intersection is in agreement with Refs. [18,19] (blue dashed lines); the harmonic waves that are not observed in the experiment are represented by dashed gray lines. The horizontal dashed gray line determines the vertical position of the fundamental wave beams intersection $Z^{*}$.

not axially symmetric about the $Z$ axis so that the wave rays located in the plane defined by the gradient to the surface and its horizontal projection do not intersect at a focal point. Instead, the intersections of these rays form a Gaussian-like curve in the $X Z$ plane with the peak located near $Z=-3$ for the rays corresponding to the second harmonic wave. Focusing of waves emitted by an oscillating elliptically shaped ridge is discussed in more detail by Bühler and Muller [24]. In contrast to the axisymmetric focusing of the fundamental waves on the side of the spheroid at $Z=0$, the focal zone of fundamental waves below the spheroid does not generate observable higher harmonics [Fig. 3(e)] due to the three-dimensional spreading of the energy over a curved focal region.

The motion observed just below the $\mathcal{H}$ spheroid, close to $Z=-1$, is generated at the frequency of the second harmonic but does not propagate; i.e., it is not observed at a larger distance from the object [Fig. 3(e)]. The second harmonic waves we observe below are emitted at the critical points and move, due to nonlinear interactions with the sheared boundary layer, along this boundary layer before taking their original direction in agreement with the dispersion relation [Fig. 3(e)]. Video recordings showed that the second harmonic wave first deviated horizontally and therefore reaches its focal point $(X, Z) \approx(0,-3)$ instead of the predicted focal point in $(X, Z)=(0,-4)$ shown in Fig. 4(a). This observation is consistent with the time-frequency results discussed in the next section.

In the right-hand column of Fig. 3, the results are shown for the vertically oriented $\mathcal{V}$ spheroid, for the same stratification, amplitude, and oscillation frequency. Again, fundamental and second harmonics are generated at critical points. For the $\mathcal{V}$ spheroid, the energy of the fundamental waves is spread along a curved zone around the spheroid instead of a focal point and therefore has a much lower amplitude. As a consequence, second harmonic waves are not observed. The sketches of the wave patterns in Figs. 4(a) and 4(b) summarize the observations in the vertical plane, showing the second harmonics (1) generated at the critical points (red dashed lines), (2) generated at in the zones of primary beam intersection (blue dashed lines), and (3) that were not observed in experiment (gray dashed lines).

\section{B. Horizontal structure}

Complementary experiments were conducted for the $\mathcal{H}$ spheroid to determine the position of generation of higher harmonics using a horizontal laser sheet at $Z_{1}=-1$ and in the zone of the primary beam intersection $Z^{*}$ [see the horizontal line in Fig. 4(a)]. The latter value, $Z^{*}$, is calculated 


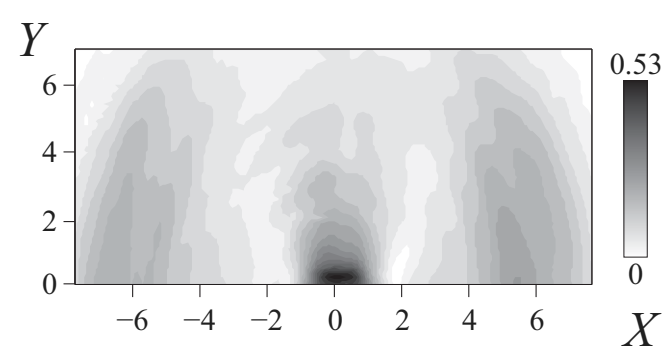

(a)

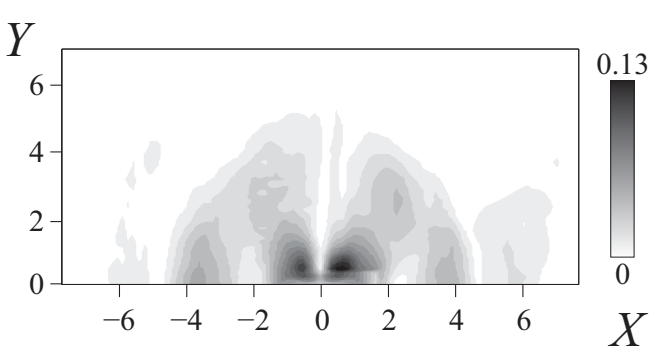

(b)

FIG. 5. Radiation patterns for the first (a) and second harmonic wave (b) of the horizontal longitudinal velocity field at the boundary $(Z=-1)$ of the oscillating $\mathcal{H}$ spheroid. Experimental parameters $A / a=$ $0.75, N=1.08 \mathrm{rad} / \mathrm{s}$, and $\Omega=0.4$.

from formula (3.14) in the linear analysis of Hurley [9]. For the sphere, the distance between the object surface and zone of primary wave beam intersection $\left(\left|Z_{1}-Z^{*}\right|=0.1\right)$ appears to be too small for PIV measurements in the horizontal plane, but for the $\mathcal{H}$ spheroid oscillating with frequency $\Omega=0.4$ this distance is large enough $\left(\left|Z_{1}-Z^{*}\right|=0.33\right)$ to visualize the flow with the laser sheet at two different positions and conduct PIV measurements.

Distributions of amplitudes of the first and second harmonics of the horizontal velocity $U_{a}$ in the horizontal plane at $Z=-1$ are presented in Fig. 5. The second harmonic close to the object $(-2<X<2)$ has a quadrupolar structure. As mentioned in the previous section, this second harmonic is generated at the critical points $\varepsilon_{2}=1$ and is affected by the shear at the boundary layer of the spheroid. The second harmonics at $|X|=4$ and $|X|=6$ correspond respectively to the outgoing wave beams generated at the critical point $\varepsilon_{2}=1$ and the wave beam generated in the zone of fundamental wave intersection at $Z=0$. Figure 6 shows that the amplitude of the second harmonic wave has its maximum close to the surface of the spheroid and decreases with the distance from the object, confirming once again the generation of higher harmonic waves according to Bell's [17] theory at the object boundary.

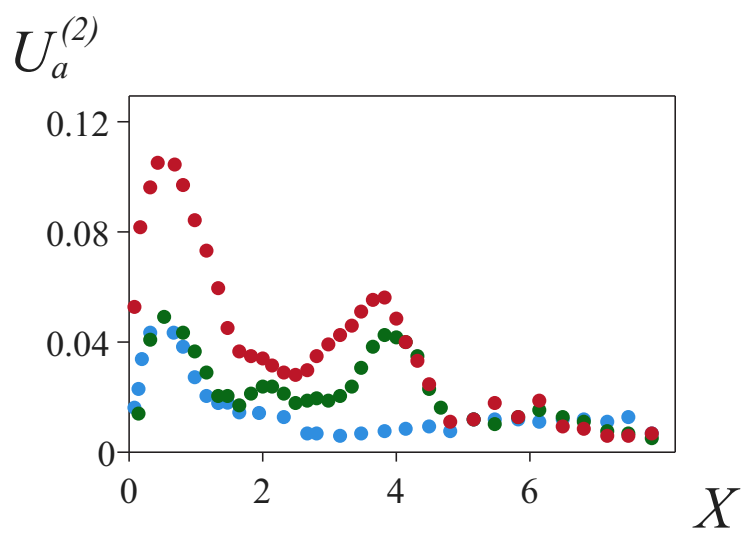

FIG. 6. Profiles of second harmonic of horizontal velocity field at $Z=-1$ (red dots), $Z=-1.47$ (green dots), and $Z=-1.91$ (blue dots) for oscillating $\mathcal{H}$ spheroid. Data obtained at $Y=0$ with $A / a=0.75, N=$ $1.08 \mathrm{rad} / \mathrm{s}$, and $\Omega=0.4$. Here the normalization $X=x / L$ is used, with the length scale $L$ in the direction of oscillation, i.e., $L=b$ in the case of the $\mathcal{H}$ spheroid. 


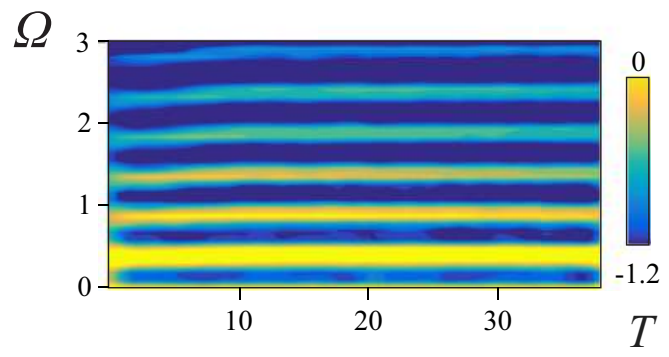

(a)

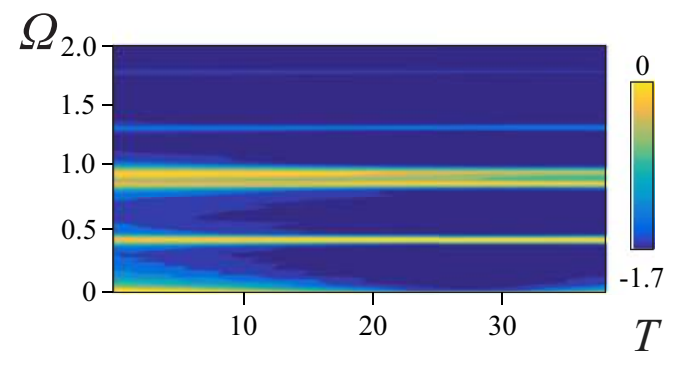

(b)

FIG. 7. Time-frequency diagram for the waves generated by the $\mathcal{H}$ spheroid, with (a) $\log _{10}\left[S_{u}(t, \omega) / S_{u}\left(t, \omega_{1}\right)\right]$ averaged over $-2<X<2,-1<Y<1$ in the horizontal plane $Z=-1$ for the horizontal longitudinal velocity $u$, experimental parameters $A / a=0.75$ and $\Omega=0.4$; and (b) $\log _{10}\left[S_{w}(t, \omega) / S_{w}\left(t, \omega_{1}\right)\right]$ measured in $-2.5<X<2.5, Y=0$ and $-4<Z<-1$ for the vertical velocity $w$, experimental parameters $A / a=0.5$ and $\Omega=0.45$.

Following the approach of Bourget et al. [25], we have determined the wave spectrum generated by the $\mathcal{H}$ spheroid and its evolution with time using a time-frequency representation described in Flandrin [20]:

$$
S_{u}(t, \omega)=\left\langle\left|\int_{-\infty}^{+\infty} u(\tau) \exp ^{-i \omega \tau} h(t-\tau) d \tau\right|^{2}\right\rangle_{x y \text { or } x z},
$$

where $h(t)$ is a Hamming window typically of a width of several oscillation periods, while the average is taken over an interrogation area $x y$ or $x z$.

Figure 7(a) shows the time-frequency diagram obtained for the logarithm of $S$ averaged in space inside a small horizontal rectangle $(-2<X<2,-1<Y<1, Z=-1)$ for the $\mathcal{H}$ spheroid oscillating at frequency $\Omega=0.4$. Under experimental conditions, the first two harmonics are propagative, with their generation starting at $t=0$, and their frequency remaining constant for all 40 oscillation periods; all higher harmonics are evanescent. The transient development of these evanescent waves is visible in Fig. 7(a) $0<T<10, \Omega>1$. Figure 7(b) shows that for the $\mathcal{H}$ spheroid oscillating at frequency $\Omega=0.45$ in addition to the second harmonic frequency $\left(\omega_{2}=0.9 \mathrm{rad} / \mathrm{s}\right)$ generated below the focal zone, waves are generated at a lower frequency $\omega_{2} \approx 0.8 \mathrm{rad} / \mathrm{s}$ above the focal zone in Fig. 3(e).

The normalized kinetic energy $E$ was calculated from the vertical and horizontal longitudinal velocity components for waves generated by the $\mathcal{H}$ spheroid in the vertical plane $Y=0$, where the transverse velocity component is 0 and averaged over $-b<X<b$ for all values between $-6<Z<-1$, i.e.,

$$
E^{(i)}=\frac{\left\langle\frac{1}{2}\left[\left(u_{a}^{(i)}\right)^{2}+\left(w_{a}^{(i)}\right)^{2}\right]\right\rangle_{x}}{\frac{1}{2}(A \omega)^{2}},
$$

with $i=1,2$ for the first and second harmonics, respectively. The distribution of averaged kinetic energy along the vertical axis $Z$ is shown in Fig. 8. The fundamental wave energy has its maximum close to the object boundary and decreases with distance $Z$. The energy of the second harmonic wave has two maxima: one close to the object $(Z=-1)$ and another one in the zone of secondary beam intersection $[Z=-2.3$; see Fig. 3(e)]. Because of the focusing, the second harmonic is dominant for $-6<Z<-3$.

Distributions of the third harmonic component of the horizontal longitudinal velocity $U_{a}^{(3)}$ and the vertical velocity $W_{a}^{(3)}$ emitted by the $\mathcal{S}$ sphere are shown in Figs. 9(a) and 9(b), respectively. The third harmonics frequency is estimated as $3 \Omega \approx 0.8$. An octupolar structure is clearly visible, 


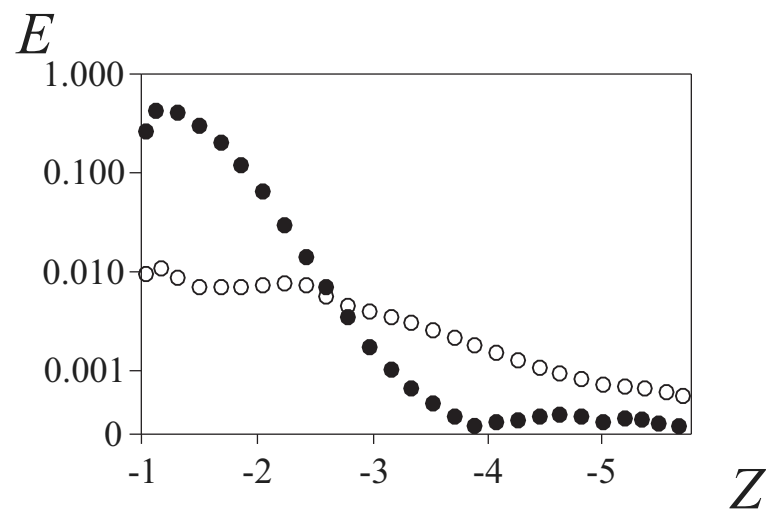

FIG. 8. Distribution of the kinetic energy $E$ defined by Eq. (3) of the first (dots) and second (circles) harmonic for $(X, Y)=(0,0)$ along $Z$. Experimental parameters: $A / a=0.5$ and $\Omega=0.45$.

with 6 azimuthal poles implying the amplitude variation as $\sin (3 \phi)$ for the horizontal velocity amplitude and $\cos (3 \phi)$ for the vertical velocity amplitude.

The distribution of the amplitudes of the third harmonic along the $X=x / L$ axis is presented in Fig. 10 for objects of different curvature: $\mathcal{S}$ sphere, $\mathcal{V}$ spheroid $(L=a)$, and $\mathcal{H}$ spheroid $(L=b)$. Dye-line visualizations showed that the fluid moves well over all obstacles, confirming that the fluid motion experienced a steeper slope for the case of the $\mathcal{V}$ spheroid. All six azimuthal poles of the third harmonic generated by the sphere have a similar form, size, and amplitude distribution but in the case of the oscillating $\mathcal{V}$ spheroid, the amplitude of the azimuthal poles along the $X$ axis is smaller compared to the amplitude of the same poles for the sphere $\left(0.4<X<1\right.$ for $U_{a}^{(3)}$ and $0.9<X<1.2$ for $\left.W_{a}^{(3)}\right)$. We notice that the structure of the wave pattern approximately scales with the horizontal radius of the object. The amplitude of the higher harmonic waves generally changes with the slope of the object, but for the $\mathcal{V}$ spheroid and $\mathcal{S}$ sphere, that possess the rotational symmetry with respect to the vertical axis, an important geometrical effect in terms of ray theory causes the waves to focus at the vertex point. For a horizontally elongated obstacle, such as the $\mathcal{H}$ spheroid, the energy focuses at a curve due to the difference of curvatures in the planes parallel to $X Z$ and $Y Z$. Thus, the focal zone (above and below the oscillating object) where the enhanced wave motions take place is not well localized for the $\mathcal{H}$ spheroid while it is well localized for the $\mathcal{S}$ sphere and $\mathcal{V}$

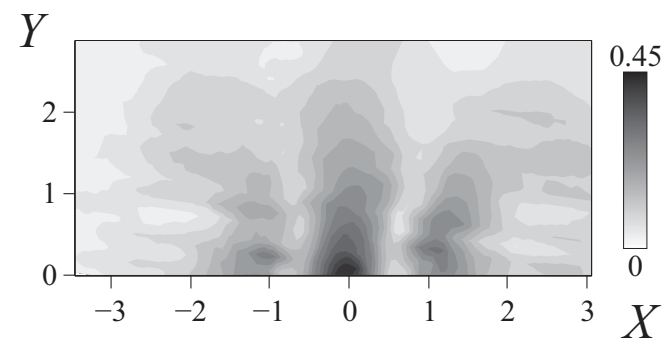

(a)

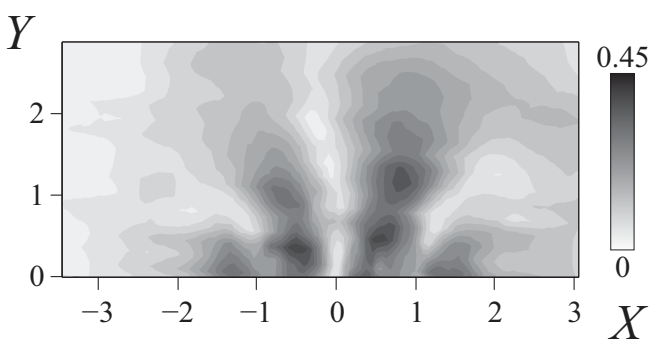

(b)

FIG. 9. Octupolar radiation pattern corresponding to the upper half of Fig. 1(c) obtained for an oscillating sphere: gray levels visualize (a) the horizontal velocity amplitude of the third harmonic wave $U_{a}^{(3)}$ and (b) the vertical velocity amplitude of the third harmonic wave $W_{a}^{(3)}$. Experimental parameters: oscillating sphere with $A / a=0.91$ at $Z=-1.66, N=1 \mathrm{rad} / \mathrm{s}, \Omega=0.27$. 


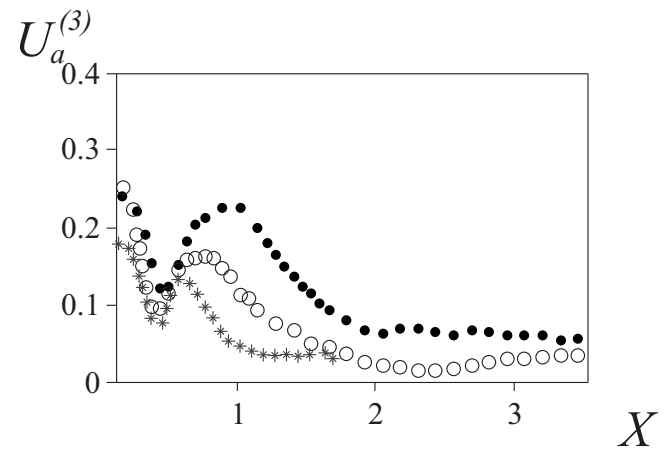

(a)

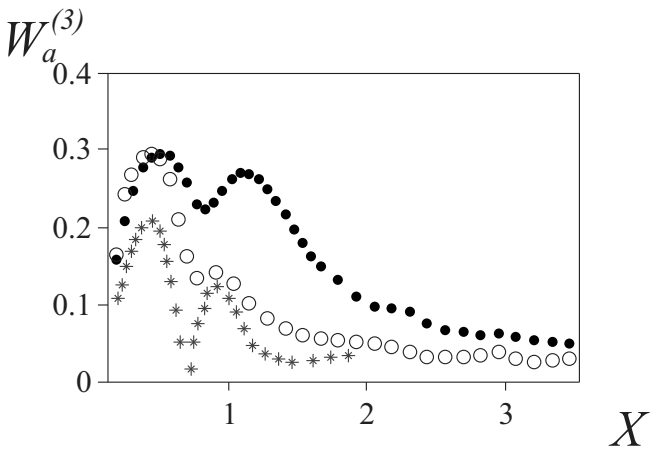

(b)

FIG. 10. Profiles of the third harmonic of (a) horizontal $\left(U_{a}^{(3)}\right)$ and (b) vertical $\left(W_{a}^{(3)}\right)$ velocity field along the $X=x / L$ axis at $Y=0$. Dots, circles, and stars correspond to results for the oscillating $\mathcal{S}$ sphere (measured at $Z=-1.66), \mathcal{V}$ spheroid $(Z=-2.4)$, and $\mathcal{H}$ spheroid $(Z=-1.66)$, respectively. The parameter $L=a$ in the cases of $\mathcal{S}$ sphere and $\mathcal{V}$ spheroid and $L=b$ in the case of $\mathcal{H}$ spheroid. Experimental parameters: $A / a=0.65, N=1 \mathrm{~s}^{-1}$, and $\Omega=0.27$. (The vertical distance to the boundary of the object is the same in all cases, but $Z$ values vary because of the scaling.)

spheroid due to their rotational symmetry. Indeed, the sphere and $\mathcal{V}$ spheroid generate waves of the same amplitude, while the slopes are different [Fig. 10; $0<X<0.4$ for $U_{a}^{(3)}$ and $0<X<0.9$ for $\left.W_{a}^{(3)}\right]$. Therefore, this focusing effect makes the principal difference between our objects. The effect of focusing internal waves have been discussed in detail by Ermanyuk et al. [26].

The dependance of extreme values of the nondimensional amplitudes of the first three harmonics on the nondimensional oscillation amplitude is presented in Fig. 11. The logarithmic representation shows that the amplitude of horizontal $\left(U_{a}\right)$ and vertical $\left(W_{a}\right)$ velocity is independent of the oscillation amplitude for the normalized fundamental wave and varies as $A / a$ and $(A / a)^{2}$ for the normalized second and third harmonics, respectively. This trend is in agreement with the two-dimensional theory of Bell [17] and previous experimental studies on fundamental and second harmonic waves generated by an oscillating sphere [15].

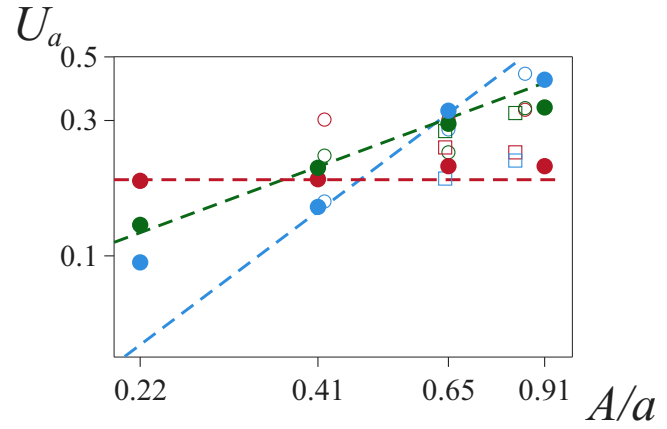

(a)

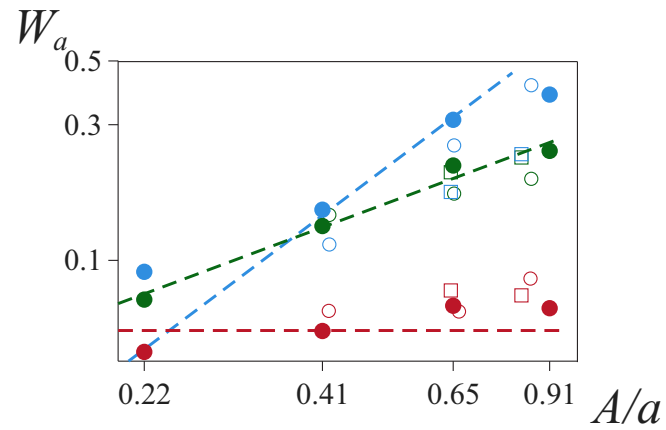

(b)

FIG. 11. Variations of the peak magnitudes of the first three harmonics with oscillation amplitude $A / a$ for (a) horizontal $\left(U_{a}\right)$ and (b) vertical $\left(W_{a}\right)$ velocity fields. First, second, and third harmonics are represented with red, green, and blue colors, respectively, and compared with theoretical predictions for the sphere (red line) and linear regressions for $U_{a}^{(2)}\left(W_{a}^{(2)}\right)$ and $U_{a}^{(3)}\left(W_{a}^{(3)}\right)$ (green and blue lines). Dots, circles, and squares correspond to results for oscillating $\mathcal{S}$ sphere, $\mathcal{V}$ spheroid, and $\mathcal{H}$ spheroid, respectively. $N=1 \mathrm{rad} / \mathrm{s}, \Omega=0.27$. 
In view of former observations on the relevance of the fundamental wave and higher harmonics [15,27], a note should be made on higher harmonics in three-dimensional flows. In the two-dimensional case, the energy content of the higher harmonics is usually relatively low, as demonstrated for the second harmonic in Zhang et al. [12]. However, in the three-dimensional case, such as for a sphere, the amplitude of the second harmonic can be higher than the amplitude of the first; they can be comparable for a Gaussian topography or a flow over a flattened topography [16]. The reason for this difference is related to the fact that, at low oscillation frequencies $\Omega$ and fixed amplitude $A / a$, the power $P$ of internal waves that are generated by horizontally oscillating bodies varies as $\Omega^{2}$ for a circular cylinder and $\Omega^{5}$ for a sphere [14], and similar asymptotic behavior is valid for elliptic cylinders and spheroids [28]. Accordingly, at low frequency $\Omega$ and fixed $A / a$, the amplitude of the first linear harmonic is much smaller in three dimensions than in two and can more easily be overtaken by the amplitude of the higher, nonlinear harmonics. Therefore, for low oscillation frequencies $\Omega$ that are relevant to geophysical applications, we may have a situation where the fundamental wave generated by barotropic flow over three-dimensional topography is weak while a higher $n$th harmonic is strong. Reciprocally, higher harmonics are of interest for three-dimensional topography because of their high energy content under certain conditions. High oscillation amplitude and frequency can be found in wave-focusing regions at a large distance from the topography. The azimuthal harmonic wave structure may have an influence on the energy and mass fluxes in the vicinity of ocean topography.

\section{CONCLUSIONS}

We have studied the generation of higher harmonic waves near oscillating boundaries of varying slope that occur for moderate to high oscillation amplitudes. Fundamental and second harmonic waves are observed at the critical points on the boundaries in agreement with the theory of Bell [17]. For high amplitude of oscillation $A / a>0.5$, overturning motions are generated in the boundary layer of a spheroid. Intersections of the fundamental wave beams lead to the generation of second harmonic waves, confirming the theoretical considerations of Tabaei et al. [18] and Jiang and Marcus [19]. However, second harmonic waves have not been observed when intersections of fundamental wave rays form a curve instead of a point. They form a ring-shaped region in the case of the $\mathcal{V}$ spheroid and a curved region below and above the $\mathcal{H}$ spheroid. The three-dimensional spreading of the energy over a larger region results in relatively weak secondary beams emitted from these zones. This could explain their absence in the observations. It is also shown that, in agreement with previous observations [15] and theoretical predictions [17], the amplitudes of the first three harmonics grow respectively linearly, quadratically, and cubically with increasing oscillation amplitudes. This geometric focusing effect is also responsible for the increase in amplitude of the waves generated by axisymmetric objects.

Our observations show that the spatial structures of the radiation patterns of the first three harmonics of the wave field are respectively dipolar, quadrupolar, and octupolar. According to this trend, we expect that the $n$th harmonic wave radiation pattern of multipolar order $2^{n}$ has $2 n$ azimuthal poles in the horizontal plane. Because of the complexity of the structure and in particular the narrowness of the wave, it is more difficult to visualize the radiation patterns of higher wave modes in small laboratory setups. In view of their relatively large amplitudes, we may expect them to be relevant also for the radiation of wave energy as generated by the tidal motion in the oceans.

\section{ACKNOWLEDGMENTS}

The authors thank B. Voisin for bringing our attention to Ref. [24], explaining the 3D geometry of the focal region and second harmonic generation. N.S. gratefully acknowledges S. Mercier and J.-M. Barnoud for their technical help and assistance with the experimental setup. N.S. thanks the French Ministry of Research for the fellowship that allowed her to conduct this work and her Ph.D. thesis at LEGI, and J.B.F. acknowledges financial support by LabEx OSUG@2020 (Investissements 
d'avenir ANR10LABX56, http://www.osug.fr/?lang=en). E.E. acknowledges support as a visiting professor at University Joseph Fourier in 2011.

[1] C. Garrett and E. Kunze, Internal tide generation in the deep ocean, Annu. Rev. Fluid Mech. 39, 57 (2007).

[2] E. G. Morozov, Semidiurnal internal wave global field, Deep-Sea Res. I 42, 135 (1995).

[3] W. Munk and C. Wunsch, Abyssal recipes II: Energetics of tidal and wind mixing, Deep-Sea Res. I 45, 1977 (1998).

[4] V. Vlasenko, N. Stashchuk, and K. Hutter, Baroclinic Tides: Theoretical Modeling and Observational Evidence (Cambridge University Press, Cambridge, UK, 2005).

[5] D. L. Boyer and X. Zhang, The interaction of time-dependent rotating and stratified flow with isolated topography, Dyn. Atmos. Oceans 14, 543 (1989).

[6] D. L. Boyer and X. Zhang, Motion of oscillatory currents past isolated topography, J. Phys. Oceanogr. 20, 1425 (1990).

[7] D. E. Mowbray and B. S. H. Rarity, A theoretical and experimental investigation of the phase configuration of internal waves of small amplitude in a density stratified liquid, J. Fluid Mech. 28, 1 (1967).

[8] D. G. Hurley and G. Keady, The generation of internal waves by vibrating elliptic cylinders, part 2: Approximate viscous solution, J. Fluid Mech. 351, 119 (1997).

[9] D. G. Hurley, The generation of internal waves by vibrating elliptic cylinders, part 1: Inviscid solution, J. Fluid Mech. 351, 105 (1997).

[10] B. R. Sutherland, S. B. Dalziel, G. O. Hughes, and P. F. Linden, Visualization and measurement of internal waves by synthetic schlieren, part 1: Vertically oscillating cylinder, J. Fluid Mech. 390, 93 (1999).

[11] B. R. Sutherland and P. F. Linden, Internal wave excitation by a vertically oscillating elliptical cylinder, Phys. Fluids 14, 721 (2002).

[12] H. P. Zhang, B. King, and H. L. Swinney, Experimental study of internal gravity waves generated by supercritical topography, Phys. Fluids 19, 096602 (2007).

[13] A. S. Korobov and K. G. Lamb, Interharmonics in internal gravity waves generated by tide-topography interaction, J. Fluid Mech. 611, 61 (2008).

[14] B. Voisin, E. V. Ermanyuk, and J.-B. Flór, Internal wave generation by oscillation of a sphere, with application to internal tides, J. Fluid Mech. 666, 308 (2011).

[15] E. V. Ermanyuk, J.-B. Flór and B. Voisin, Spatial structure of first and higher harmonic internal waves from a horizontally oscillating sphere, J. Fluid Mech. 671, 364 (2011).

[16] B. King, H. P. Zhang, and H. L. Swinney, Tidal flow over three-dimensional topography generates out-of-forcing-plane harmonics, Geophys. Res. Lett. 37, L14606 (2010).

[17] T. H. Bell, Lee waves in stratified flows with simple harmonic time dependance, J. Fluid Mech. 67, 705 (1975).

[18] A. Tabaei, T. R. Akylas, and K. G. Lamb, Nonlinear effects in reflecting and colliding internal wave beams, J. Fluid Mech. 526, 217 (2005).

[19] C.-H. Jiang and P. S. Marcus, Selection Rules for the Nonlinear Interaction of Internal Gravity Waves, Phys. Rev. Lett. 102, 124502 (2009).

[20] P. Flandrin, Time-Frequency/Time-Scale Analysis (Academic Press, San Diego, CA, 1998).

[21] M. J. Mercier, N. B. Garnier, and T. Dauxois, Reflection and diffraction of internal waves analyzed with the Hilbert transform, Phys. Fluids 20, 086601 (2008).

[22] A. Fincham and G. Delerce, Advanced optimization of correlation imaging velocimetry algorithms, Exp. Fluids 29, S013 (2000).

[23] T. Peacock and P. Weidman, The effect of rotation on conical wave beams in a stratified fluid, Exp. Fluids 39, 32 (2005).

[24] O. Bühler and C. J. Muller, Instability and focusing of internal tides in the deep ocean, J. Fluid Mech. 588, 1 (2007). 
[25] B. Bourget, T. Dauxois, S. Joubaud, and P. Odier, Experimental study of parametric subharmonic instability for internal plane waves, J. Fluid Mech. 723, 1 (2013).

[26] E. V. Ermanyuk, N. D. Shmakova, and J.-B. Flór, Internal wave focusing by a horizontally oscillating torus, J. Fluid Mech. 813, 695 (2017).

[27] S. J. Ghaemsaidi and T. Peacock, 3D Stereoscopic PIV visualization of the axisymmetric conical internal wave field generated by an oscillating sphere, Exp. Fluids 54, 1454 (2013).

[28] E. V. Ermanyuk, The rule of affine similitude for the force coefficients of a body oscillating in a uniformly stratified fluid, Exp. Fluids 32, 242 (2002). 